\title{
Discrete Dirac Structures and Implicit Discrete Lagrangian and Hamiltonian Systems
}

\author{
Melvin Leok* and Tomoki Ohsawa ${ }^{\dagger}$ \\ *Department of Mathematics, University of California, San Diego, La Jolla, CA 92093-0112, USA \\ ${ }^{\dagger}$ Department of Mathematics, University of Michigan, Ann Arbor, MI 48109-1043, USA
}

\begin{abstract}
We present discrete analogues of Dirac structures and the Tulczyjew's triple by considering the geometry of symplectic maps and their associated generating functions. We demonstrate that this framework provides a means of deriving discrete analogues of implicit Lagrangian and Hamiltonian systems. In particular, this yields implicit nonholonomic Lagrangian and Hamiltonian integrators. We also introduce discrete Lagrange-d'Alembert-Pontryagin and Hamilton-d'Alembert variational principles, which provide an alternative derivation of the same set of integration algorithms. In addition to providing a unified treatment of discrete Lagrangian and Hamiltonian mechanics in the more general setting of Dirac mechanics, it provides a generalization of symplectic and Poisson integrators to the broader category of Dirac integrators.
\end{abstract}

Keywords: Dirac structures, discrete mechanics, Lagrangian mechanics, Hamiltonian mechanics. PACS: $02.40 . \mathrm{Yy}, 45.10 . \mathrm{Db}, 45.10 . \mathrm{Na}, 45.20 . \mathrm{Jj}$

\section{INTRODUCTION}

Dirac structures, which can be viewed as simultaneous generalizations of symplectic and Poisson structures, were introduced in Courant [1, 2]. In the context of geometric mechanics [3-5], Dirac structures are of interest as they can directly incorporate Dirac constraints that arise in degenerate Lagrangian systems [6], LC circuits [7-9], interconnected systems [10], and nonholonomic systems [11], and thereby provide a unified geometric framework for studying such problems.

From the Hamiltonian perspective, these systems are described by implicit Hamiltonian systems [8, 12]. An implicit Hamiltonian system is defined by a Hamiltonian and a Dirac structure, which is a subbundle that satisfies certain conditions. On the Lagrangian side, an implicit Lagrangian system [9] is defined by exploiting the geometric structure called the Tulczyjew's triple [13, 14] in addition to a Dirac structure.

In this paper, we introduce discrete analogues of induced Dirac structures [9] and the Tulczyjew's triple, which provide the natural setting to define implicit discrete Lagrangian and Hamiltonian systems. We show that they recover nonholonomic integrators [15, 16] that are typically derived from a discrete Lagrange-d'Alembert principle.

We also introduce discrete Lagrange-d'Alembert-Pontryagin and Hamiltond'Alembert variational principles, that provide a variational characterization of implicit discrete Lagrangian and Hamiltonian systems that were described using discrete Dirac structures, and which reduce to the standard Lagrangian [17] and Hamiltonian [18, 19] variational integrators in the absence of constraints. Discrete Lagrangian, Hamiltonian, and nonholonomic mechanics have also been generalized to Lie groupoids [20-23]. 


\section{DIRAC STRUCTURES, TULCZYJEW'S TRIPLE, AND IMPLICIT LAGRANGIAN SYSTEMS}

We first briefly review induced Dirac structures, which give rise to implicit Lagrangian systems (ILS) [See 9, 24, 25]. Implicit Lagrangian systems are particularly useful in formulating systems with degenerate Lagrangians and/or constraints.

\subsection{Induced Dirac Structures and Tulczyjew's Triple}

The key idea in formulating implicit Lagrangian systems for systems with constraints is to introduce a Dirac structure that is induced by the constraints. A particularly important class of Dirac structures is the induced Dirac structure on a cotangent bundle defined in the following way: Let $Q$ be a manifold, $\pi_{Q}: T^{*} Q \rightarrow Q$ be the cotangent bundle projection, and $\Omega^{b}: T T^{*} Q \rightarrow T^{*} T^{*} Q$ be the flat map associated with the standard symplectic structure $\Omega$ on $T^{*} Q$.

Proposition 1 ([8, 9, 12]). Let $\Delta_{Q}$ be a constant-dimensional distribution on $Q$. Define $\Delta_{T^{*} Q}:=\left(T \pi_{Q}\right)^{-1}\left(\Delta_{Q}\right) \subset T T^{*} Q$ and let $\Delta_{T^{*} Q}^{\circ} \subset T^{*} T^{*} Q$ be its annihilator. Then,

$$
D_{\Delta_{Q}}:=\left\{(v, \alpha) \in T T^{*} Q \oplus T^{*} T^{*} Q \mid v \in \Delta_{T^{*} Q} \text { and } \alpha-\Omega^{b}(v) \in \Delta_{T^{*} Q}^{\circ}\right\} .
$$

is an almost Dirac structure.

Following Tulczyjew [13, 14] and Yoshimura and Marsden [9], let us first introduce the Tulczyjew's triple, i.e., the diffeomorphisms $\Omega^{b}, \kappa_{Q}$, and $\gamma_{Q}:=\Omega^{b} \circ \kappa_{Q}^{-1}$ between the iterated tangent and cotangent bundles:

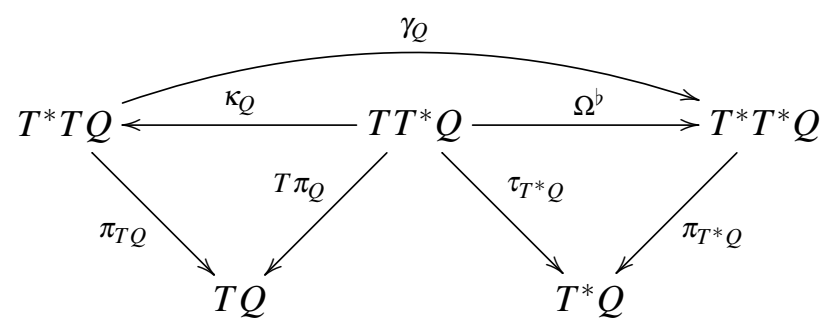

or in local coordinates

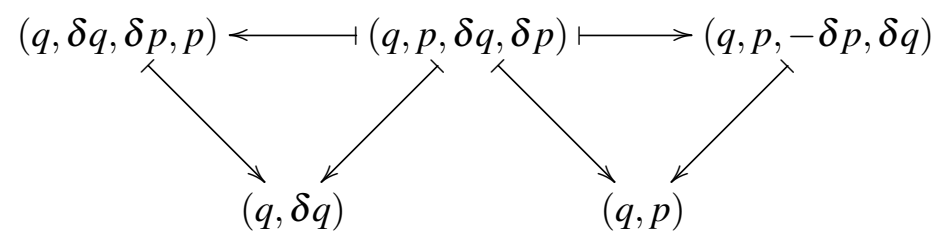

The maps $\Omega^{b}$ and $\kappa_{Q}$ induce symplectic forms on $T T^{*} Q$ in the following way: Let $\Theta_{T^{*} T^{*} Q}$ and $\Theta_{T^{*} T Q}$ be standard Lagrange one-forms on the cotangent bundles $T^{*} T^{*} Q$ and $T^{*} T Q$, respectively. Then, one defines one-forms $\chi$ and $\lambda$ on $T T^{*} Q$ by pullback,

$$
\chi:=\left(\Omega^{b}\right)^{*} \Theta_{T^{*} T^{*} Q}=-\delta p d q+\delta q d p, \quad \lambda:=\left(\kappa_{Q}\right)^{*} \Theta_{T^{*} T Q}=\delta p d q+p d(\delta q) .
$$


Then, these one-forms induce the two-form $\Omega_{T T^{*} Q}$ on $T T^{*} Q$ by

$$
\Omega_{T T^{*} Q}:=-d \lambda=d \chi=d q \wedge d(\delta p)+d(\delta q) \wedge d p
$$

which is a symplectic form on $T T^{*} Q$. In particular,

$$
\Omega_{T T^{*} Q}=\left(\kappa_{Q}\right)^{*} \Omega_{T^{*} T Q}=-\left(\Omega^{b}\right)^{*} \Omega_{T^{*} T^{*} Q},
$$

where $\Omega_{T^{*} T Q}$ and $\Omega_{T^{*} T^{*} Q}$ are the canonical symplectic structures on $T^{*} T Q$ and $T^{*} T^{*} Q$, respectively.

\subsection{Implicit Lagrangian and Hamiltonian Systems}

To define an implicit Lagrangian system, it is necessary to introduce the Dirac differential of a Lagrangian function: Given a Lagrangian $L: T Q \rightarrow \mathbb{R}$, we define the Dirac differential $\mathfrak{D L}: T Q \rightarrow T^{*} T^{*} Q$ by

$$
\mathfrak{D} L:=\gamma_{Q} \circ d L
$$

In local coordinates,

$$
\mathfrak{D} L(q, v)=\left(q, \frac{\partial L}{\partial v},-\frac{\partial L}{\partial q}, v\right)
$$

We are now ready to define an implicit Lagrangian system:

Definition 2 (Implicit Lagrangian/Hamiltonian Systems). Suppose that a Lagrangian $L: T Q \rightarrow \mathbb{R}$ and a Dirac structure $D \subset T T^{*} Q \oplus T^{*} T^{*} Q$ are given. Let $X \in \mathfrak{X}\left(T^{*} Q\right)$ be a partial vector field on $T^{*} Q$, defined at points of $P:=\mathbb{F} L\left(\Delta_{Q}\right) \subset T^{*} Q$. Then an implicit Lagrangian system (ILS) is defined by

$$
(X, \mathfrak{D} L) \in D \text {. }
$$

In particular, if $D$ is the induced Dirac structure $D_{\Delta_{Q}}$ given in Eq. (1), Eq. (8) reduces to

$$
T \pi_{Q}(X) \in \Delta_{Q}, \quad \Omega^{b}(X)-\mathfrak{D} L \in \Delta_{T^{*} Q}^{\circ},
$$

or in local coordinates, by setting $X=\dot{q} \partial_{q}+\dot{p} \partial_{p}$,

$$
\dot{q} \in \Delta_{Q}, \quad \dot{q}=v, \quad p=\frac{\partial L}{\partial v}, \quad \dot{p}-\frac{\partial L}{\partial q} \in \Delta_{Q}^{\circ} .
$$

Similarly, with a Hamiltonian $H: T^{*} Q \rightarrow \mathbb{R}$, an implicit Hamiltonian system (IHS) [8, [12] is defined by

$$
(X, d H) \in D .
$$

In particular, with $D=D_{\Delta_{Q}}$, Eq. (11) reduces to

$$
T \pi_{Q}(X) \in \Delta_{Q}, \quad \Omega^{b}(X)-d H \in \Delta_{T^{*} Q}^{\circ},
$$

or in local coordinates, by setting $X=\dot{q} \partial_{q}+\dot{p} \partial_{p}$,

$$
\dot{q} \in \Delta_{Q}, \quad \dot{q}=\frac{\partial H}{\partial p}, \quad \dot{p}+\frac{\partial H}{\partial q} \in \Delta_{Q}^{\circ} .
$$




\section{DISCRETE ANALOGUES OF TULCZYJEW'S TRIPLE}

In this section we construct discrete analogues of the Tulczyjew's triple shown in Eq. (2) that retain the key geometric properties, especially the symplecticity of the maps involved. This makes it possible to formulate a natural discrete analogue of implicit Lagrangian and Hamiltonian systems that gives a structure-preserving discretization of these systems. The discussion is limited to the case where the configuration space $Q$ is a vector space.

\subsection{Generating Functions and the Maps $\kappa_{Q}^{d}, \Omega_{d \pm}^{b}$}

The guiding principle here is to make use of the properties of generating functions, instead of smooth symplectic flows. The idea behind it is the observation made by Lall and West [18] that the discrete Lagrangian and Hamiltonians are essentially generating functions of type 1, 2, and 3 (using the terminology set by Goldstein et al. [26]).

Let us first review some basic facts about generating functions. Consider a map $F: T^{*} Q \rightarrow T^{*} Q$ written as $\left(q_{0}, p_{0}\right) \mapsto\left(q_{1}, p_{1}\right)$. Note that, since $Q$ is assumed to be a vector space here, the cotangent bundle is trivial, i.e., $T^{*} Q \cong Q \times Q^{*}$, and so one can write $F: Q \times Q^{*} \rightarrow Q \times Q^{*}$ as well. One then considers the following three maps associated with $F$ :

(i) $F_{1}: Q \times Q \rightarrow Q^{*} \times Q^{*} ;\left(q_{0}, q_{1}\right) \mapsto\left(p_{0}, p_{1}\right)$,

(ii) $F_{2}: Q \times Q^{*} \rightarrow Q^{*} \times Q ;\left(q_{0}, p_{1}\right) \mapsto\left(p_{0}, q_{1}\right)$,

(iii) $F_{3}: Q^{*} \times Q \rightarrow Q \times Q^{*} ;\left(p_{0}, q_{1}\right) \mapsto\left(q_{0}, p_{1}\right)$.

The generating function of type $i$ (with $i=1,2,3$ ) is a scalar function $S_{i}$ defined on the range of the map $F_{i}$ that exists if and only if the map $F$ is symplectic.

We first relate the generating function of type 1 with a discrete analogue of the map $\kappa_{Q}$ in the Tulczyjew's triple in Eq. (2). We regard $\left(p_{0}, p_{1}\right)$ as functions of $\left(q_{0}, q_{1}\right)$ as indicated in the definition of the map $F_{1}$ above, and define $i_{F_{1}}: Q \times Q \rightarrow T^{*} Q \times T^{*} Q$ by

$$
i_{F_{1}}:\left(q_{0}, q_{1}\right) \mapsto\left(\left(q_{0}, p_{0}\right),\left(q_{1}, p_{1}\right)\right) \text { where }\left(p_{0}, p_{1}\right)=F_{1}\left(q_{0}, q_{1}\right) .
$$

Recall that the map $F:\left(q_{0}, p_{0}\right) \mapsto\left(q_{1}, p_{1}\right)$ is symplectic if and only if $d q_{0} \wedge d p_{0}=$ $d q_{1} \wedge d p_{1}$ or equivalently $d\left(-p_{0} d q_{0}+p_{1} d q_{1}\right)=0$. By the Poincaré lemma, this is equivalent to the existence of a function $S_{1}: Q \times Q \rightarrow \mathbb{R}$, a generating function of type 1 , such that

$$
-p_{0} d q_{0}+p_{1} d q_{1}=d S_{1}\left(q_{0}, q_{1}\right) \Longleftrightarrow p_{0}=-D_{1} S_{1}\left(q_{0}, q_{1}\right), \quad p_{1}=D_{2} S_{1}\left(q_{0}, q_{1}\right) .
$$

This yields the map $\kappa_{Q}^{d}: T^{*} Q \times T^{*} Q \rightarrow T^{*}(Q \times Q)$ so that the diagram below commutes.
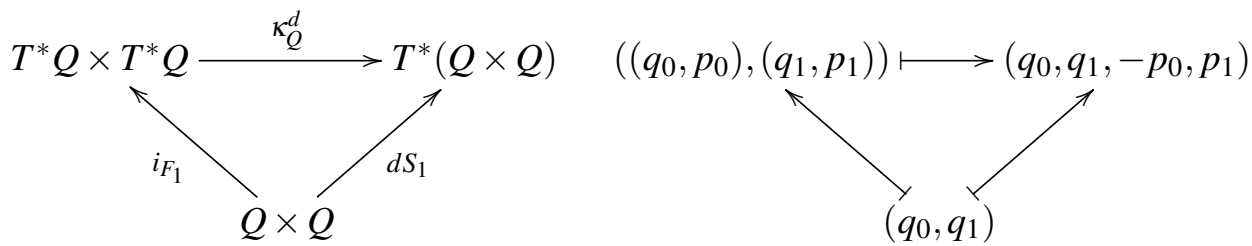
Similar arguments applied to the maps $F_{2}$ and $F_{3}$ and type 2 and 3 generating functions $S_{2}: Q \times Q^{*} \rightarrow \mathbb{R}$ and $S_{3}: Q^{*} \times Q \rightarrow \mathbb{R}$ give rise to the maps $\Omega_{d+}^{b}: T^{*} Q \times T^{*} Q \rightarrow$ $T^{*}\left(Q \times Q^{*}\right)$ and $\Omega_{d-}^{b}: T^{*} Q \times T^{*} Q \rightarrow T^{*}\left(Q^{*} \times Q\right)$ so that the diagrams below commute.
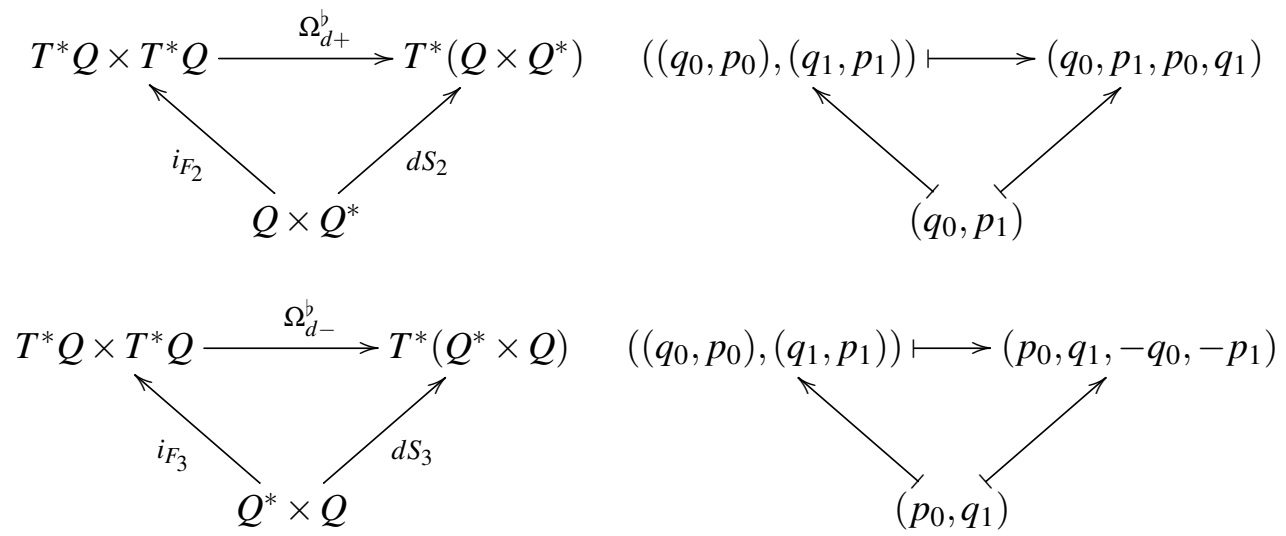

\subsection{Discrete Tulczyjew Triples}

Combining the diagrams in Eqs. (14) and (15), we obtain

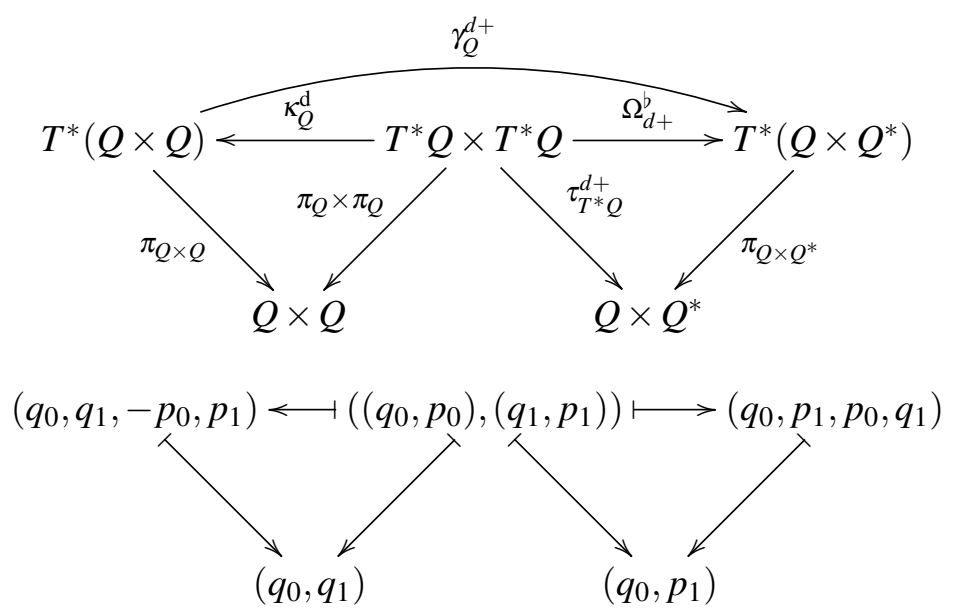

We call the diagram the $(+)$-discrete Tulczyjew triple. The reason for the plus sign will become clear later.

The maps $\kappa_{Q}^{d}$ and $\Omega_{d+}^{b}$ inherit the properties of $\kappa_{Q}$ and $\Omega^{b}$ discussed in Section 2.1 in the following sense: Let $\Theta_{T^{*}\left(Q \times Q^{*}\right)}$ and $\Theta_{T^{*}}(Q \times Q)$ be the Lagrange one-forms on $Q \times Q^{*}$ and $T^{*}(Q \times Q)$, respectively. The maps $\kappa_{Q}^{d}$ and $\Omega_{d+}^{b}$ induce two Lagrange one-forms on $T^{*} Q \times T^{*} Q$. One is

$$
\chi_{d+}:=\left(\Omega_{d+}^{b}\right)^{*} \Theta_{T^{*}\left(Q \times Q^{*}\right)}=p_{0} d q_{0}+q_{1} d p_{1},
$$

and the other is

$$
\lambda_{d+}:=\left(\kappa_{Q}^{d}\right)^{*} \Theta_{T^{*}(Q \times Q)}=-p_{0} d q_{0}+p_{1} d q_{1} .
$$


These one-forms induce the two-form $\Omega_{T^{*} Q \times T^{*} Q}$ by

$$
\Omega_{T^{*} Q \times T^{*} Q}=-d \lambda_{d+}=d \chi_{d+}=d q_{1} \wedge d p_{1}-d q_{0} \wedge d p_{0} .
$$

Combining the diagrams in Eqs. (14) and (16), we obtain

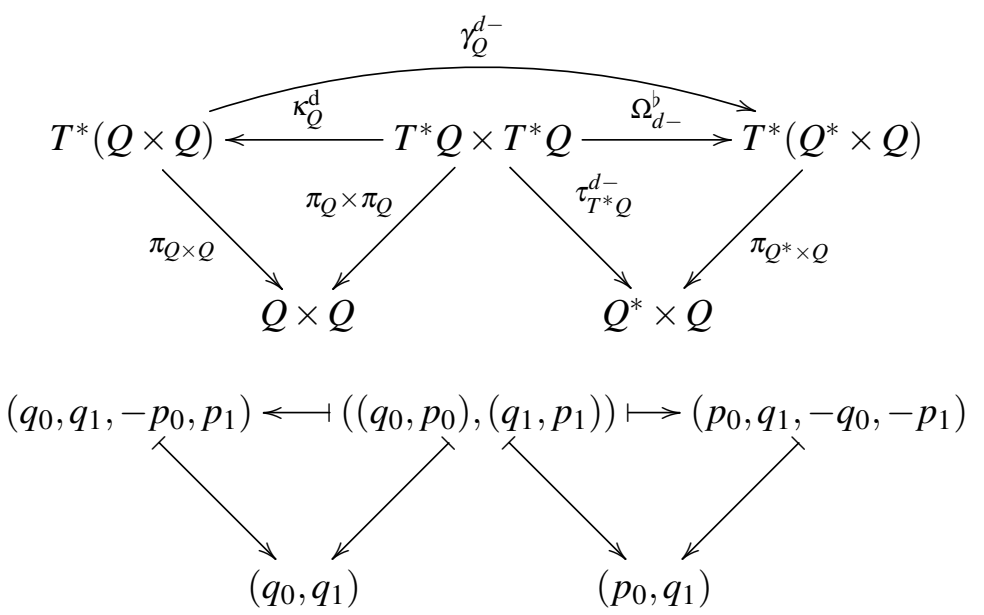

We call the diagram the (-)-discrete Tulczyjew triple. Again, the reason for the minus sign will become clear later.

As in the $(+)$-discrete case, we can reinterpret the maps $\kappa_{Q}^{d}$ and $\Omega_{d-}^{b}$ as follows: Let $\Theta_{T^{*}\left(Q^{*} \times Q\right)}$ be the symplectic one-form on $Q^{*} \times Q$. Then, we have

$$
\chi_{d-}:=\left(\Omega_{d-}^{b}\right)^{*} \Theta_{T^{*}\left(Q^{*} \times Q\right)}=-p_{1} d q_{1}-q_{0} d p_{0},
$$

and

$$
\lambda_{d-}:=\left(\kappa_{Q}^{d}\right)^{*} \Theta_{T^{*}(Q \times Q)}=-p_{0} d q_{0}+p_{1} d q_{1},
$$

and they induce the same symplectic form $\Omega_{T^{*} Q \times T^{*} Q}$ as above:

$$
\Omega_{T^{*} Q \times T^{*} Q}:=-d \lambda_{d-}=d \chi_{d-}=d q_{1} \wedge d p_{1}-d q_{0} \wedge d p_{0}
$$

Then, we have,

$$
\Omega_{T^{*} Q \times T^{*} Q}=\left(\kappa_{Q}^{d}\right)^{*} \Omega_{T^{*}(Q \times Q)}=-\left(\Omega_{d+}^{b}\right)^{*} \Omega_{T^{*}\left(Q \times Q^{*}\right)}=-\left(\Omega_{d-}^{b}\right)^{*} \Omega_{T^{*}\left(Q^{*} \times Q\right)},
$$

where $\Omega_{T^{*}(Q \times Q)}, \Omega_{T^{*}\left(Q \times Q^{*}\right)}$ and $\Omega_{T^{*}\left(Q^{*} \times Q\right)}$ are the canonical symplectic structures on $T^{*}(Q \times Q), T^{*}\left(Q \times Q^{*}\right)$ and $T^{*}\left(Q^{*} \times Q\right)$, respectively.

\section{DISCRETE ANALOGUES OF INDUCED DIRAC STRUCTURES}

Recall from Section 2.1 that, given a constraint distribution $\Delta_{Q} \subset T Q$, we first defined the distribution $\Delta_{T^{*} Q} \subset T T^{*} Q$ and then constructed the induced Dirac structure $D_{\Delta_{Q}} \subset$ $T T^{*} Q \oplus T^{*} T^{*} Q$. This section introduces a discrete analogue of this construction. 


\subsection{Discrete constraint distributions}

Since the tangent bundle $T Q$ is replaced by the product $Q \times Q$ in the discrete setting, a natural discrete analogue of a constraint distribution $\Delta_{Q} \subset T Q$ is a subset $\Delta_{Q}^{d} \subset Q \times Q$. Cortés and Martínez [15] (see also McLachlan and Perlmutter [16]) proposed a way to construct discrete constraints $\Delta_{Q}^{d} \subset Q \times Q$ based on given (continuous) constraints $\Delta_{Q} \subset T Q$. Let us briefly summarize their approach here: First introduce the annihilator of $\Delta_{Q}$, that is denoted by $\Delta_{Q}^{\circ} \subset T^{*} Q$ and is defined, for each $q \in Q$, as

$$
\Delta_{Q}^{\circ}(q):=\left\{\alpha_{q} \in T_{q}^{*} Q \mid \forall v_{q} \in \Delta_{Q},\left\langle\alpha_{q}, v_{q}\right\rangle=0\right\} .
$$

Let $m:=\operatorname{dim} Q-\operatorname{dim} \Delta_{Q}$. Then one can find a basis of $m$ constraint one-forms $\left\{\omega^{i}\right\}_{i=1}^{m}$ for the annihilator subspace:

$$
\Delta_{Q}^{\circ}=\operatorname{span}\left\{\omega^{a}\right\}_{a=1}^{m}
$$

By using the one-forms $\omega^{a}$, and a retraction $\tau: T Q \rightarrow Q \times Q$, one defines functions $\omega_{d}^{a}: Q \times Q \rightarrow \mathbb{R}$, by $\omega_{d}^{a}:=\omega^{a} \circ \tau^{-1}$, whose annihilation defines the discrete constraint set:

$$
\Delta_{Q}^{d}:=\left\{\left(q_{0}, q_{1}\right) \in Q \times Q \mid \omega_{d}^{a}\left(q_{0}, q_{1}\right)=0, a=1,2, \ldots, m\right\} .
$$

Next we need to introduce a discrete analogue of the $\Delta_{T^{*} Q}$ using the discrete constraint $\Delta_{Q}^{d}$ defined above. A natural discrete analogue of $\Delta_{T^{*} Q}$ would be $\Delta_{T^{*} Q}^{d} \subset T^{*} Q \times T^{*} Q$ defined by

$$
\Delta_{T^{*} Q}^{d}:=\left(\pi_{Q} \times \pi_{Q}\right)^{-1}\left(\Delta_{Q}^{d}\right)=\left\{\left(\left(q_{0}, p_{0}\right),\left(q_{1}, p_{1}\right)\right) \in T^{*} Q \times T^{*} Q \mid\left(q_{0}, q_{1}\right) \in \Delta_{Q}^{d}\right\} .
$$

We will also need the corresponding annihilator distributions on $Q \times Q^{*}$ and $Q^{*} \times Q$, or in other words, discrete analogues of $\Delta_{T^{*}}^{\circ}$. To this end, we first introduce the projections

$$
\begin{aligned}
& \pi_{T^{*} Q}^{d+}: T^{*}\left(Q \times Q^{*}\right) \rightarrow T^{*} Q ;\left(q, p, \alpha_{q}, \alpha_{p}\right) \mapsto\left(q, \alpha_{q}\right), \\
& \pi_{T^{*} Q}^{d-}: T^{*}\left(Q^{*} \times Q\right) \rightarrow T^{*} Q ;\left(p, q, \alpha_{p}, \alpha_{q}\right) \mapsto\left(q, \alpha_{q}\right) .
\end{aligned}
$$

Then we define the annihilator distributions $\Delta_{Q \times Q^{*}}^{\circ} \subset T^{*}\left(Q \times Q^{*}\right)$ and $\Delta_{Q^{*} \times Q}^{\circ} \subset T^{*}\left(Q^{*} \times\right.$ $Q)$; more explicitly, we have

$$
\begin{aligned}
& \Delta_{Q \times Q^{*}}^{\circ}:=\left(\pi_{T^{*} Q}^{d+}\right)^{-1}\left(\Delta_{Q}^{\circ}\right)=\left\{\left(q, p, \alpha_{q}, 0\right) \in T^{*}\left(Q \times Q^{*}\right) \mid \alpha_{q} \in \Delta_{Q}^{\circ}(q)\right\}, \\
& \Delta_{Q^{*} \times Q}^{\circ}:=\left(\pi_{T^{*} Q}^{d-}\right)^{-1}\left(\Delta_{Q}^{\circ}\right)=\left\{\left(p, q, 0, \alpha_{q}\right) \in T^{*}\left(Q^{*} \times Q\right) \mid \alpha_{q} \in \Delta_{Q}^{\circ}(q)\right\} .
\end{aligned}
$$

\subsection{Discrete Induced Dirac Structures}

Now we are ready to define discrete analogues of the induced Dirac structures $D_{\Delta_{Q}}$ shown in Proposition 1 .

Definition 3 (Discrete Induced Dirac Structures). Given a discrete constraint distribution $\Delta_{Q}^{d} \subset Q \times Q$, define the (+)-discrete induced Dirac structure $D_{\Delta_{Q}}^{d+} \subset\left(T^{*} Q \times T^{*} Q\right) \times$ 
$T^{*}\left(Q \times Q^{*}\right)$ by

$$
\begin{aligned}
D_{\Delta_{Q}}^{d+}:=\left\{\left(\left(z, z^{+}\right), \alpha_{\hat{z}}\right) \in\left(T^{*} Q \times T^{*} Q\right) \times T^{*}\left(Q \times Q^{*}\right) \mid\right. \\
\left.\left(z, z^{+}\right) \in \Delta_{T^{*} Q}^{d}, \alpha_{\hat{z}}-\Omega_{d+}^{b}\left(\left(z, z^{+}\right)\right) \in \Delta_{Q \times Q^{*}}^{\circ}\right\}
\end{aligned}
$$

where if $z=(q, p)$ and $z^{+}=\left(q^{+}, p^{+}\right)$then $\hat{z}:=\left(q, p^{+}\right)$.

Likewise, define the (-)-discrete Induced Dirac structure by

$$
\begin{aligned}
D_{\Delta_{Q}}^{d-}:=\left\{\left(\left(z^{-}, z\right), \alpha_{\tilde{z}}\right) \in\left(T^{*} Q \times T^{*} Q\right) \times T^{*}\left(Q^{*} \times Q\right) \mid\right. \\
\left.\left(z^{-}, z\right) \in \Delta_{T^{*} Q}^{d}, \alpha_{\tilde{z}}-\Omega_{d-}^{b}\left(\left(z^{-}, z\right)\right) \in \Delta_{Q^{*} \times Q}^{\circ}\right\},
\end{aligned}
$$

where if $z=(q, p)$ and $z^{-}=\left(q^{-}, p^{-}\right)$then $\tilde{z}:=\left(p^{-}, q\right)$.

\section{DISCRETE DIRAC MECHANICS}

Now that we have discrete analogues of both the Tulczyjew's triple and the induced Dirac structures at our disposal, we are ready to define discrete analogues of implicit Lagrangian and Hamiltonian systems. As we shall see, two types of implicit discrete Lagrangian/Hamiltonian systems will follow from the $( \pm)$-discrete Tulczyjew triple and induced $( \pm)$-Dirac structures.

\section{1. (+)-Discrete Dirac Mechanics}

Let us first introduce a discrete analogue of the Dirac differential: Let $\gamma_{Q}^{d+}:=\Omega_{d+}^{b}$ 。 $\left(\kappa_{Q}^{d}\right)^{-1}: T^{*}(Q \times Q) \rightarrow T^{*}\left(Q \times Q^{*}\right)$, and for a given discrete Lagrangian $L_{d}: Q \times Q \rightarrow \mathbb{R}$, define $\mathfrak{D}^{+} L_{d}:=\gamma_{Q}^{d+} \circ d L_{d}$.

Definition 4 (Implicit (+)-Discrete Lagrangian/Hamiltonian System). Suppose that a discrete Lagrangian $L_{d}: Q \times Q \rightarrow \mathbb{R}$ and a discrete constraint distribution $\Delta_{Q}^{d} \subset Q \times Q$ are given. Let $X_{d}^{k}=\left(\left(q_{k}, p_{k}\right),\left(q_{k+1}, p_{k+1}\right)\right) \in T^{*} Q \times T^{*} Q$ be a discrete analogue of a vector field on $T^{*} Q$. Then, an implicit $(+)$-discrete Lagrangian system is a triple $\left(L_{d}, \Delta_{Q}^{d}, X_{d}\right)$ with

$$
\left(X_{d}^{k}, \mathfrak{D}^{+} L_{d}\left(q_{k}, q_{k}^{+}\right)\right) \in D_{\Delta_{Q}}^{d+} .
$$

In coordinates, we have the following $(+)$-discrete implicit Lagrange-d'Alembert equations:

$$
\begin{gathered}
\left(q_{k}, q_{k+1}\right) \in \Delta_{Q}^{d}, \quad q_{k+1}=q_{k}^{+}, \\
p_{k+1}=D_{2} L_{d}\left(q_{k}, q_{k}^{+}\right), \quad p_{k}+D_{1} L_{d}\left(q_{k}, q_{k}^{+}\right) \in \Delta_{Q}^{\circ}\left(q_{k}\right) .
\end{gathered}
$$


Similarly, with a right discrete Hamiltonian [18] $H_{d+}: Q \times Q^{*} \rightarrow \mathbb{R}$, an implicit $(+)$ discrete Hamiltonian system is a triple $\left(H_{d+}, \Delta_{Q}^{d}, X_{d}\right)$ with

$$
\left(X_{d}^{k}, d H_{d+}\left(q_{k}, p_{k+1}\right)\right) \in D_{\Delta_{Q}}^{d+} .
$$

In coordinates, we have the following $(+)$-discrete implicit Hamilton equations:

$$
\left(q_{k}, q_{k+1}\right) \in \Delta_{Q}^{d}, \quad q_{k+1}=D_{2} H_{d+}\left(q_{k}, p_{k+1}\right), \quad p_{k}-D_{1} H_{d+}\left(q_{k}, p_{k+1}\right) \in \Delta_{Q}^{\circ}\left(q_{k}\right) .
$$

\section{2. (-)-Discrete Dirac Mechanics}

Let us first introduce the $(-)$-version of the Dirac differential: Let $\gamma_{Q}^{d-}:=\Omega_{d-}^{b} \circ$ $\left(\kappa_{Q}^{d}\right)^{-1}: T^{*}(Q \times Q) \rightarrow T^{*}\left(Q^{*} \times Q\right)$, and for a given discrete Lagrangian $L_{d}: Q \times Q \rightarrow \mathbb{R}$, define $\mathfrak{D}^{-} L_{d}:=\gamma_{Q}^{d-} \circ d L_{d}$.

Definition 5 (Implicit (-)-Discrete Lagrangian/Hamiltonian Systems). Suppose that a discrete Lagrangian $L_{d}: Q \times Q \rightarrow \mathbb{R}$ and a discrete constraint distribution $\Delta_{Q}^{d} \subset Q \times Q$ are given. Let $X_{d}^{k}=\left(\left(q_{k}, p_{k}\right),\left(q_{k+1}, p_{k+1}\right)\right) \in T^{*} Q \times T^{*} Q$ be a discrete analogue of a vector field on $T^{*} Q$. Then an implicit (-)-discrete Lagrangian system is a triple $\left(L_{d}, \Delta_{Q}^{d}, X_{d}\right)$ with

$$
\left(X_{d}^{k}, \mathfrak{D}^{-} L_{d}\left(q_{k+1}^{-}, q_{k+1}\right)\right) \in D_{\Delta_{Q}}^{d-} .
$$

In coordinates, we have the following (-)-discrete implicit Lagrange-d'Alembert equations:

$$
\begin{gathered}
\left(q_{k}, q_{k+1}\right) \in \Delta_{Q}^{d}, \quad q_{k}=q_{k+1}^{-}, \\
p_{k}=-D_{1} L_{d}\left(q_{k+1}^{-}, q_{k+1}\right), \quad p_{k+1}-D_{2} L_{d}\left(q_{k+1}^{-}, q_{k+1}\right) \in \Delta_{Q}^{\circ}\left(q_{k+1}\right) .
\end{gathered}
$$

Similarly, with a left discrete Hamiltonian [18] $H_{d-}: Q^{*} \times Q \rightarrow \mathbb{R}$, an implicit (-)discrete Hamiltonian system is a triple $\left(H_{d-}, \Delta_{Q}^{d}, X_{d}\right)$ with

$$
\left(X_{d}^{k}, d H_{d-}\left(p_{k}, q_{k+1}\right)\right) \in D_{\Delta_{Q}}^{d-}
$$

In coordinates, we have the following (-)-discrete implicit Hamilton equations:

$$
\left(q_{k}, q_{k+1}\right) \in \Delta_{Q}^{d}, \quad q_{k}=-D_{1} H_{d-}\left(p_{k}, q_{k+1}\right), \quad p_{k+1}+D_{2} H_{d-}\left(p_{k}, q_{k+1}\right) \in \Delta_{Q}^{\circ}\left(q_{k+1}\right) .
$$

\section{VARIATIONAL STRUCTURE FOR IMPLICIT LAGRANGIAN AND HAMILTONIAN SYSTEMS}

In this section we come back to the continuous setting discussed in Section 2 briefly to discuss variational formulations of the implicit Lagrangian and Hamiltonian systems 
again following Yoshimura and Marsden [24]. We will develop the corresponding discrete analogues in the next section.

Definition 6. Suppose that a Lagrangian $L: T Q \rightarrow \mathbb{R}$ and a constraint distribution $\Delta_{Q} \subset T Q$ are given. The Lagrange-d'Alembert-Pontryagin principle is the augmented variational principle on the Pontryagin bundle $T Q \oplus T^{*} Q$ defined by

$$
\delta \int_{a}^{b}[L(q, v)+p(\dot{q}-v)] d t=0,
$$

where we impose $\dot{q} \in \Delta_{Q}$ and also the second-order curve condition $\dot{q}=v$ using Lagrange multipliers $p \in T^{*} Q$; furthermore, the variations $\delta q$ are assumed to satisfy the constraints, i.e., $\delta q \in \Delta_{Q}$, and also to vanish at the endpoints $\delta q(a)=\delta q(b)=0$.

Definition 7. Suppose that a Hamiltonian $H: T^{*} Q \rightarrow \mathbb{R}$ and a constraint distribution $\Delta_{Q} \subset T Q$ are given. The Hamilton-d'Alembert principle in phase space is the augmented variational principle defined by

$$
\delta \int_{a}^{b}[p \dot{q}-H(q, p)] d t=0
$$

where we impose the condition $\dot{q} \in \Delta_{Q}$; also the variations $\delta q$ are assumed to satisfy the constraints, i.e., $\delta q \in \Delta_{Q}$, and also to vanish at the endpoints $\delta q(a)=\delta q(b)=0$.

Proposition 8 (Yoshimura and Marsden [24]). The implicit Lagrangian system Eq. (10) and the implicit Hamiltonian system Eq. (13) follow from the Lagrange-d'AlembertPontryagin and Hamilton-d'Alembert principle in phase space, respectively.

\section{DISCRETE VARIATIONAL STRUCTURE FOR IMPLICIT DISCRETE LAGRANGIAN AND HAMILTONIAN SYSTEMS}

This section develops discrete analogues of the variational structure discussed in the last section. It is shown that the discrete versions of Lagrange-d'Alembert-Pontryagin principle and Hamilton-d'Alembert principle in phase space yield implicit discrete Lagrangian and Hamiltonian systems, respectively.

Definition $9((+)$-Discrete Lagrange-d'Alembert-Pontryagin Principle). Suppose that a discrete Lagrangian $L_{d}: Q \times Q \rightarrow \mathbb{R}$, a constraint distribution $\Delta_{Q} \subset T Q$, and a discrete constraint distribution $\Delta_{Q}^{d} \subset Q \times Q$ are given. Then the $(+)$-discrete Lagranged'Alembert-Pontryagin principle is the discrete augmented variational principle defined by

$$
\delta \sum_{k=0}^{N-1}\left[L_{d}\left(q_{k}, q_{k}^{+}\right)+p_{k+1}\left(q_{k+1}-q_{k}^{+}\right)\right]=0,
$$

where we impose $\left(q_{k}, q_{k+1}\right) \in \Delta_{Q}^{d}$ and also the discrete second-order curve condition $q_{k+1}=q_{k}^{+}$using Lagrange multipliers $p_{k+1} \in Q^{*}$; furthermore, the variations $\left(\delta q_{k}, \delta q_{k}^{+}, \delta p_{k+1}\right)$ of $\left(q_{k}, q_{k}^{+}, p_{k+1}\right) \in(Q \times Q) \oplus\left(Q \times Q^{*}\right) \cong Q \times Q \times Q^{*}$ (identifying the first $Q$ of each) are assumed to satisfy $\delta q_{k} \in \Delta_{Q}\left(q_{k}\right)$, and also to vanish at the endpoints $\delta q_{0}=\delta q_{N}=0$. 
Definition 10 ((-)-Discrete Lagrange-d'Alembert-Pontryagin Principle). Under the same setting as above, the (-)-discrete Lagrange-d'Alembert-Pontryagin principle is the discrete augmented variational principle defined by

$$
\delta \sum_{k=0}^{N-1}\left[L_{d}\left(q_{k+1}^{-}, q_{k+1}\right)-p_{k}\left(q_{k}-q_{k+1}^{-}\right)\right]=0,
$$

where we impose $\left(q_{k}, q_{k+1}\right) \in \Delta_{Q}^{d}$ and also the discrete second-order curve condition $q_{k}=q_{k+1}^{-}$using Lagrange multipliers $p_{k} \in Q^{*}$; furthermore, the variations $\left(\delta q_{k}^{-}, \delta p_{k}, \delta q_{k+1}\right)$ of $\left(q_{k}^{-}, p_{k}, q_{k+1}\right) \in(Q \times Q) \oplus\left(Q^{*} \times Q\right) \cong Q \times Q^{*} \times Q$ (identifying the last $Q$ of each) are assumed to satisfy $\delta q_{k+1} \in \Delta_{Q}\left(q_{k+1}\right)$, and also to vanish at the endpoints $\delta q_{0}=\delta q_{N}=0$.

Proposition 11. The $( \pm)$-discrete Lagrange-d'Alembert-Pontryagin principles yield the implicit $( \pm)$-discrete Lagrange-d'Alembert equations (37) and (41), respectively.

Proof. See Leok and Ohsawa [27].

Definition $12((+)$-Discrete Hamilton-d'Alembert Principle in Phase Space). Suppose that a right discrete Hamiltonian $H_{d+}: Q \times Q^{*} \rightarrow \mathbb{R}$, a constraint distribution $\Delta_{Q} \subset T Q$, and a discrete constraint distribution $\Delta_{Q}^{d} \subset Q \times Q$ are given. Then the $(+)$-discrete Hamilton-d'Alembert principle in phase space is the discrete augmented variational principle defined by

$$
\delta \sum_{k=0}^{N-1}\left[p_{k+1} q_{k+1}-H_{d+}\left(q_{k}, p_{k+1}\right)\right]=0
$$

where we impose $\left(q_{k}, q_{k+1}\right) \in \Delta_{Q}^{d}$; furthermore, the variations $\left(\delta q_{k}, \delta p_{k+1}\right)$ of $\left(q_{k}, p_{k+1}\right) \in Q \times Q^{*}$ are assumed to satisfy the constraints $\delta q_{k} \in \Delta_{Q}$, and also to vanish at the endpoints $\delta q_{0}=\delta q_{N}=0$.

Definition $13((-)$-Discrete Hamilton-d'Alembert Principle in Phase Space). Suppose that a left discrete Hamiltonian $H_{d-}: Q^{*} \times Q \rightarrow \mathbb{R}$, a constraint distribution $\Delta_{Q} \subset T Q$, and a discrete constraint distribution $\Delta_{Q}^{d} \subset Q \times Q$ are given. Then the (-)-discrete Hamilton-d'Alembert principle in phase space is the discrete augmented variational principle defined by

$$
\delta \sum_{k=0}^{N-1}\left[-p_{k} q_{k}-H_{d-}\left(p_{k}, q_{k+1}\right)\right]=0
$$

where we impose $\left(q_{k}, q_{k+1}\right) \in \Delta_{Q}^{d}$; furthermore, the variations $\left(\delta p_{k}, \delta q_{k+1}\right)$ of $\left(p_{k}, q_{k+1}\right) \in Q^{*} \times Q$ are assumed to satisfy the constraints $\delta q_{k} \in \Delta_{Q}$, and also to vanish at the endpoints $\delta q_{0}=\delta q_{N}=0$.

Proposition 14. The $( \pm)$-discrete Hamilton-d'Alembert principles yield the implicit $( \pm)$-discrete Hamilton equations (39) and (43), respectively.

Proof. See Leok and Ohsawa [27]. 


\section{ACKNOWLEDGMENTS}

We gratefully acknowledge helpful comments and suggestions of Jerrold Marsden, Joris Vankerschaver, and the referees. This material is based upon work supported by the National Science Foundation under the applied mathematics grant DMS-0726263 and the Faculty Early Career Development (CAREER) award DMS-1010687.

\section{REFERENCES}

1. T. Courant, Transactions of the American Mathematical Society 319, 631-661 (1990).

2. T. Courant, Journal of Physics A: Mathematical and General 23, 5153-5168 (1990).

3. V. I. Arnold, Mathematical Methods of Classical Mechanics, Springer, 1989.

4. R. Abraham, and J. E. Marsden, Foundations of Mechanics, Addison-Wesley, 1978, 2nd edn.

5. J. E. Marsden, and T. S. Ratiu, Introduction to Mechanics and Symmetry, Springer-Verlag, 1999.

6. Künzle, Annales de l'institut Henri Poincaré (A) 11, 393-414 (1969).

7. A. M. Bloch, and P. E. Crouch, "Representations of Dirac Structures on Vector Spaces and Nonlinear L-C Circuits," in Differential Geometry and Control Theory, American Mathematical Society, 1997, pp. 103-117.

8. A. J. van der Schaft, Reports on Mathematical Physics 41, 203-221 (1998).

9. H. Yoshimura, and J. E. Marsden, Journal of Geometry and Physics 57, 133-156 (2006).

10. A. J. van der Schaft, "Port-Hamiltonian systems: an introductory survey," in Proceedings of the International Congress of Mathematicians, 2006, vol. 3.

11. A. M. Bloch, Nonholonomic Mechanics and Control, Springer-Verlag, 2003.

12. M. Dalsmo, and A. J. van der Schaft, SIAM Journal on Control and Optimization 37, 54-91 (1998).

13. W. M. Tulczyjew, C. R. Acad. Sc. Paris 283, 15-18 (1976).

14. W. M. Tulczyjew, C. R. Acad. Sc. Paris 283, 675-678 (1976).

15. J. Cortés, and S. Martínez, Nonlinearity 14, 1365-1392 (2001).

16. R. McLachlan, and M. Perlmutter, Journal of Nonlinear Science 16, 283-328 (2006).

17. J. E. Marsden, and M. West, Acta Numerica pp. 357-514 (2001).

18. S. Lall, and M. West, Journal of Physics A: Mathematical and General 39, 5509-5519 (2006).

19. M. Leok, and J. Zhang, Preprint (arXiv: 1001.140 ) (2010).

20. A. Weinstein, "Lagrangian mechanics and groupoids," in Mechanics day (Waterloo, ON, 1992), Amer. Math. Soc., Providence, RI, 1996, vol. 7 of Fields Inst. Commun., pp. 207-231.

21. J. C. Marrero, D. Martín de Diego, and E. Martínez, Nonlinearity 19, 1313-1348 (2006).

22. D. Iglesias, J. C. Marrero, D. M. Martín de Diego, and E. Martínez, J. Nonlinear Sci. 18, $221-276$ (2008).

23. A. Stern, J. Symplectic Geom. (arXiv:0905.4318) (2010), to appear.

24. H. Yoshimura, and J. E. Marsden, Journal of Geometry and Physics 57, 209-250 (2006).

25. H. Yoshimura, and J. E. Marsden, "Dirac Structures and the Legendre Transformation for Implicit Lagrangian and Hamiltonian Systems," in Lagrangian and Hamiltonian Methods for Nonlinear Control 2006, 2007, pp. 233-247.

26. H. Goldstein, C. P. Poole, and J. L. Safko, Classical Mechanics, Addison Wesley, 2001, 3rd edn.

27. M. Leok, and T. Ohsawa, Preprint (arXiv: 0810 . 0740) (2008). 